\section{Interaction of noncoding RNA with the rDNA promoter mediates recruitment of DNMT3b and silencing of rRNA genes}

\author{
Kerstin-Maike Schmitz, ${ }^{1}$ Christine Mayer, ${ }^{1}$ \\ Anna Postepska, and Ingrid Grummt ${ }^{2}$
}

Division of Molecular Biology of the Cell II, German Cancer Research Center, DKFZ-ZMBH Alliance, D-69120 Heidelberg, Germany

Noncoding RNAs are important components of regulatory networks controlling the epigenetic state of chromatin. We analyzed the role of pRNA (promoterassociated RNA), a noncoding RNA that is complementary to the rDNA promoter, in mediating de novo CpG methylation of rRNA genes (rDNA). We show that pRNA interacts with the target site of the transcription factor TTF-I, forming a DNA:RNA triplex that is specifically recognized by the DNA methyltransferase DNMT3b. The results reveal a compelling new mechanism of RNA-dependent DNA methylation, suggesting that recruitment of DNMT3b by DNA:RNA triplexes may be a common and generally used pathway in epigenetic regulation.

Supplemental material is available at http://www.genesdev.org.

Received April 28, 2010; revised version accepted August 26, 2010.

Noncoding RNAs (ncRNAs) play a profound and complex role in regulating gene expression (Goodrich and Kugel 2009). While the mechanistic details of how RNA and chromatin are connected remain unclear, there is increasing evidence that epigenetic regulation likely represents a balanced interplay of both RNA and chromatin fields (Bernstein and Allis 2005; Mattick 2009). Previous studies have established that ncRNA plays a key role in epigenetic silencing of rRNA genes. Mammalian genomes contain several clusters of tandemly arrayed rRNA genes (rDNA), of which only a subset is transcribed. Silent rDNA repeats are marked by heterochromatic histone modifications and CpG methylation of the rDNA promoter (Santoro et al. 2002). The establishment and maintenance of the heterochromatic state is mediated by NoRC (Strohner et al. 2001), a chromatin remodeling complex comprising the ATPase SNF2h and a large subunit, termed TIP5 (TTF-Iinteracting protein \#5). NoRC silences rRNA genes by recruiting enzymes that mediate heterochromatin formation

[Keywords: RNA polymerase I; noncoding RNA; DNA methylation; DNA:RNA triplexes; DNMTs]

${ }^{1}$ These authors contributed equally to this work.

${ }^{2}$ Corresponding author.

E-MAIL i.grummt@dkfz.de; FAX 49-6221-423404.

Article is online at http://www.genesdev.org/cgi/doi/10.1101/gad.590910. and silencing (Zhou et al. 2002; Zhou and Grummt 2005). Transcriptional silencing involves DNA methylation at a critical CpG residue (CpG-133) within the upstream control element (UCE) of the rDNA promoter, thereby impairing binding of the transcription factor UBF and abrogating transcription complex formation (Santoro and Grummt 2001). Importantly, NoRC function requires the association of TIP5 with RNA that originates from an RNA polymerase I (Pol I) promoter located in the intergenic spacer $\sim 2 \mathrm{~kb}$ upstream of the pre-rRNA transcription start site (Mayer et al. 2006). These intergenic transcripts are of low abundance and usually do not accumulate in vivo because they are rapidly degraded or processed into 150 - to 250 -nucleotide (nt) RNAs that are shielded from degradation by binding to NoRC. These NoRC-associated transcripts are termed pRNA (for promoter-associated RNA), as their sequence matches the rDNA promoter. TIP5 recognizes the secondary structure of pRNA, and the interaction of TIP5 with pRNA changes the structure of both pRNA and NoRC in an induced fit mechanism (Mayer et al. 2008). Antisensemediated depletion of pRNA leads to nucleoplasmic distribution of NoRC, hypomethylation of rDNA, and enhanced Pol I transcription. "RNA refeeding" assays involving a brief incubation of pRNA-depleted cells with synthetic transcripts revealed that the nucleolar localization of NoRC could be restored by exogenous pRNA (Mayer et al. 2008). pRNA folds into a conserved secondary structure that is required for binding to TIP5 and targeting NoRC to nucleoli (Mayer et al. 2008). Antisense-mediated depletion of pRNA leads to displacement of NoRC from nucleoli, decrease in rDNA methylation, and activation of Pol I transcription, underscoring the essential role of pRNA in epigenetic silencing of rRNA genes.

In this study, we investigated the role of ectopic pRNA in triggering de novo DNA methylation and transcriptional silencing. We show that elevated levels of synthetic pRNA trigger de novo DNA methylation, heterochromatin formation, and transcriptional silencing. The results demonstrate that a stretch of $20 \mathrm{nt}$ in pRNA interacts with $\mathrm{T}_{0}$, the binding site for the transcription factor TTF-I, possibly forming a triple-stranded structure that is recognized by DNMT3b. The results reveal a compelling mechanism by which a ncRNA can target DNA methyltransferase (DNMT) to a specific genomic site, thereby inducing DNA methylation and transcriptional silencing.

\section{Results and Discussion}

To examine the role of pRNA in epigenetic regulation of rRNA genes, we elevated cellular pRNA levels by transfecting pRNA-205/-1, a synthetic transcript covering rDNA promoter sequences from -205 to -1 , into NIH3T3 cells and monitored DNA methylation, heterochromatin formation, and pre-rRNA levels. Consistent with previous results, PCR analysis of genomic DNA digested with the methylation-sensitive enzyme HpaII and bisulfite sequencing revealed that ectopic pRNA triggered de novo methylation of the rDNA promoter, increasing from $\sim 40 \%$ in mock-transfected NIH3T3 cells to $\sim 70 \%$ in cells transfected with pRNA-205/-1 (Supplemental Fig. S1A,B). Increased DNA methylation correlated with elevation of heterochromatic histone modifications at the rDNA promoter (such as H4K20me3 and 
H3K27me3), decrease of euchromatic histone marks (such as H3K4me2) (Supplemental Fig. S1C), and reduction of cellular pre-rRNA levels (Supplemental Fig. S1A).

pRNA folds into a conserved stem-loop structure (nucleotides from -127 to -39 ) that is required for binding to TIP5 and nucleolar localization of NoRC (Mayer et al. 2008). Therefore, pRNA lacking sequences required for stem-loop formation (pRNA-113/-39) does not mediate de novo DNA methylation and transcriptional silencing (Fig. 1A). Unexpectedly, pRNA-127/39 did not trigger DNA methylation and transcriptional silencing, even though it contained the sequence that folds into the hairpin structure required for interaction with TIP5 (Fig. 1A). Replacement of sequences upstream of the TIP5-binding domain by unrelated sequences also disturbed the function of pRNA (pRNA/LS-205/-127). Thus, although the stem-loop structure of pRNA is necessary and sufficient for TIP5 binding and heterochromatin formation, de novo DNA methylation requires 5 '-terminal pRNA sequences covering the UCE and $T_{0}$,

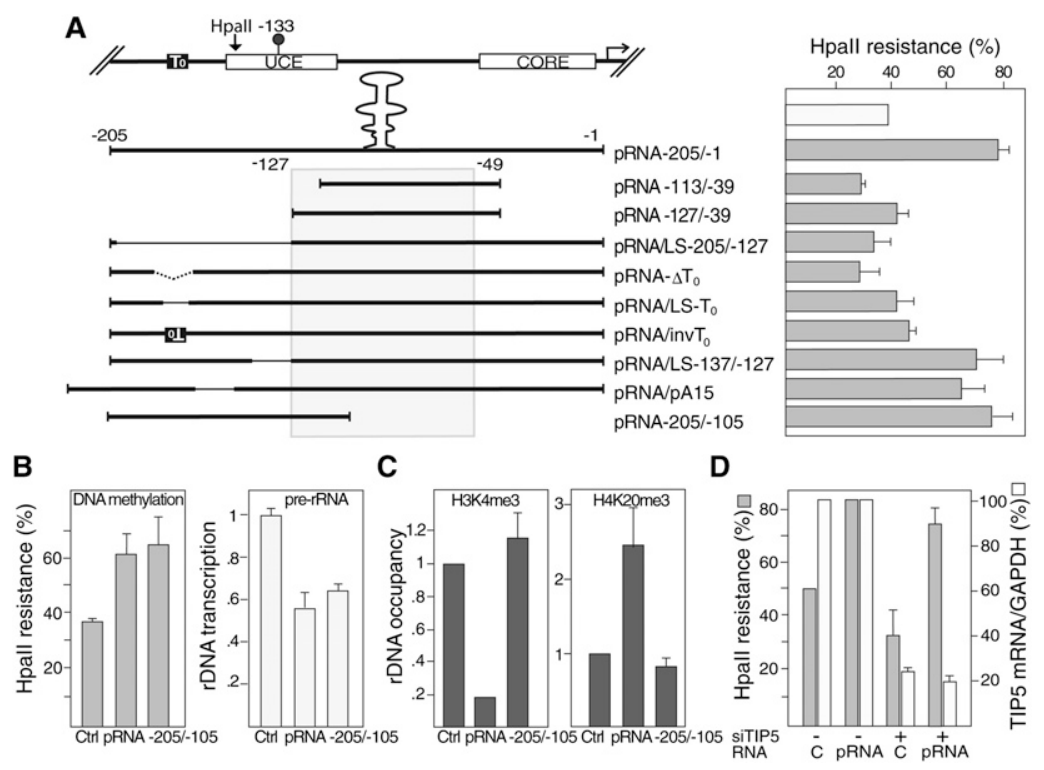

Figure 1. Ectopic pRNA mediates DNA methylation and transcriptional silencing. (A) Schematic display of the rDNA promoter and synthetic pRNAs used. The $\mathrm{T}_{0}$ element (black box), the UCE, the core promoter (CORE), the CpG residue at -133 (lollipop) and the HpaII site at -143 are marked. The minimal TIP5-binding domain of pRNA is shaded in gray, and a cartoon depicting the stem-loop structure of pRNA is shown above. The numbers refer to the position of nucleotides with respect to the transcription start site. The thick lines represent pRNA, and the thin lines indicate unrelated RNA. (Right) The bar diagram summarizes the capability of the respective pRNA derivatives to mediate de novo methylation of the rDNA promoter. NIH3T3 cells were transfected with control RNA (light bar) or the indicated pRNAs, and rDNA methylation was monitored by methylation-sensitive restriction analysis $(n=$ 5). (B) Upstream pRNA sequences are sufficient to mediate de novo methylation of the rDNA promoter. NIH3T3 cells were transfected with control RNA (Ctrl), pRNA205/-1 (pRNA), or pRNA-205/-105 (205/-105). rDNA methylation was analyzed by methylation-sensitive PCR (gray bars), and pre-rRNA levels were monitored by RTqPCR (light bars; $n=4$ ). (C) pRNA-205/-1 but not pRNA-205/-105 mediates heterochromatin formation at the rDNA promoter. Cells were transfected as in $B$ and rDNA promoter occupancy of $\mathrm{H} 3 \mathrm{~K} 4 \mathrm{me} 3$ and $\mathrm{H} 4 \mathrm{~K} 20 \mathrm{me} 3$ was monitored by chromatin immunoprecipitation (ChIP) $(n=3)$. $(D)$ pRNA mediates NoRC-independent rDNA methylation. NIH3T3 cells that have been transfected with off-target siRNA (-) or siRNA against TIP5 were retransfected after $96 \mathrm{~h}$ with 140 pmol of control RNA (C) or synthetic pRNA-205/-1 (pRNA), and methylation of the rDNA promoter was analyzed by methylation-sensitive qPCR. The efficiency of TIP5 knockdown was monitored by RT-qPCR (light bars). the promoter-proximal binding site for the transcription factor TTF-I (Grummt et al. 1986). Deletion of $\mathrm{T}_{0}$ pRNA- $\left.\Delta \mathrm{T}_{0}\right)$, randomization of the $\mathrm{T}_{0}$ sequence (pRNA/

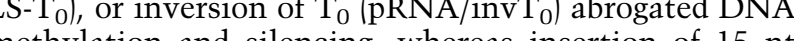
$\mathrm{T}_{0}$ are required for de novo methylation of the rDNA

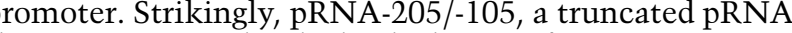
DA and transcriptional silencing (Fig. 1A,B). However f the $205 /-105$ was incapable of triggering the removal menting the heterochromatic histone mark H4K20me3, ectopic pRNA mediates rDNA hypermethylation in the of NoRC, we transfected TIP5-depleted cells with pRNA-205/-1 and analyzed rDNA methylation. Overexpression of pRNA enhanced DNA methylation, even if TIP5 was depleted (Fig. 1D). Thus, pRNA on its own triggers methylation of the rDNA promoter, provided that it contains sequences matching $\mathrm{T}_{0}$. This suggests that $\mathrm{pRNA}$ cooperates with $\mathrm{T}_{0}$ in guiding DNMT(s) to rDNA to methylate CpG-133, which, if methylated, impairs binding of UBF and transcription complex assembly (Santoro and Grummt 2001).

To examine whether pRNA interacts with $\mathrm{T}_{0}$ to guide DNMT(s) to the rDNA promoter, we cotransfected cells with biotin-labeled pRNA and with rDNA reporter plasmids that contain or lack $\mathrm{T}_{0}$, respectively, and captured pRNA-associated plasmid DNA on streptavidincoated beads. As shown in Figure 2A, biotinylated pRNA was associated with the wild-type reporter plasmid harboring $\mathrm{T}_{0}(\mathrm{pMr} 1930)$ but not with the plasmid lacking $\mathrm{T}_{0}\left(\mathrm{pMr} 1930 \Delta \mathrm{T}_{0}\right)$, emphasizing the critical role of $\mathrm{T}_{0}$ in targeting pRNA to nucleolar chromatin. Likewise, endogenous rDNA was captured if cells were transfected with a biotinylated oligoribonucleotide comprising $\mathrm{T}_{0}$ (oligo \#4) but not with a control oligoribonucleotide (oligo \#1). A similar enrichment of captured rDNA was observed if isolated nuclei were incubated with biotinylated oligo \#4, emphasizing the requirement of $T_{0}$ in targeting pRNA to nucleolar chromatin.

Consistent with binding of pRNA to chromatin requiring $T_{0}$ sequences, chromatin RNA immunoprecipitation (ChRIP) assays revealed that endogenous pRNA is bound to chromatin that is associated with NoRC and heterochromatic histone modifications but not to genes carrying euchromatic histone marks (Fig. 2B). Consistently, pRNA mutants lacking $\mathrm{T}_{0}$ (pRNA- $\Delta \mathrm{T}_{0}$ and pRNA-127/-39) were not associated with heterochromatin (Fig. 2C). To exclude the possibility that pRNA is tethered to chromatin by interaction with TTF-I or other proteins, we modified the 
A

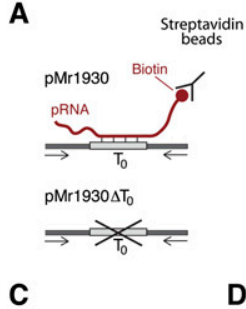

C

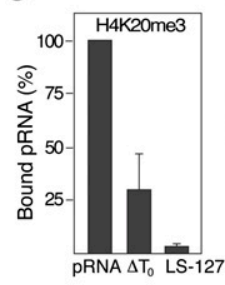

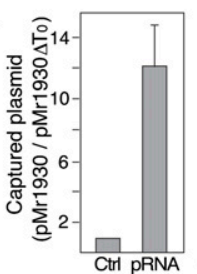

D
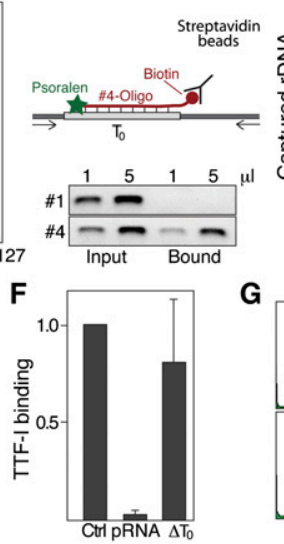

G
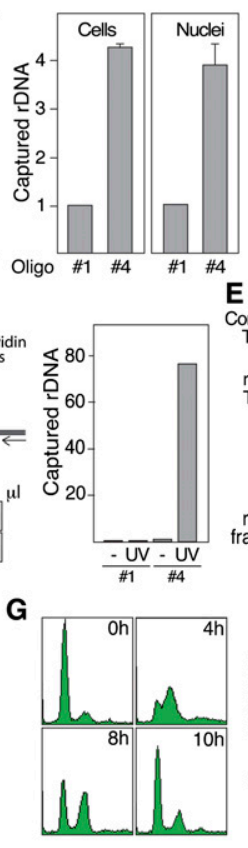

B

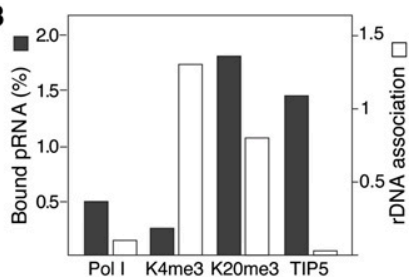

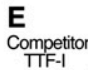
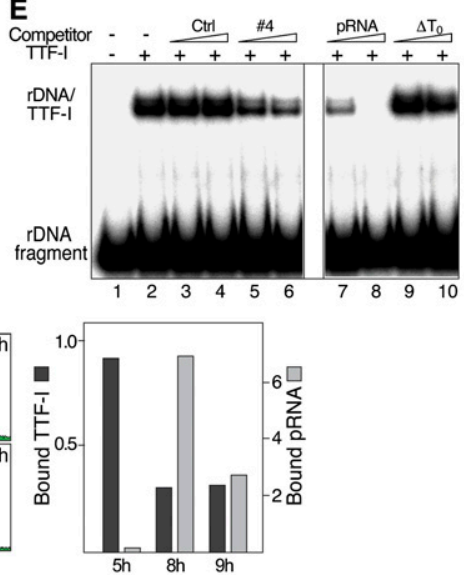

Figure 2. pRNA interacts with $\mathrm{T}_{0}$ in vivo. (A) Triplex capture assay. (Left) NIH3T3 cells were cotransfected with the rDNA reporter plasmids pMr1930 or pMr1930 $\Delta \mathrm{T}_{0}$ and biotin-labeled control RNA (Ctrl) or pRNA-205/-1. Cell lysates were incubated with streptavidin-coated beads and captured DNA was analyzed by qPCR. Data are presented as the ratio of captured pMr1930 versus pMr1930 $\Delta \mathrm{T}_{0}$. (Right) Triplex capture assay using cross-linked chromatin from cells that were transfected with biotinylated oligo \#1 or oligo \#4 (Cells), or from nuclei that were preincubated with $10 \mu \mathrm{M}$ biotinylated oligoribonucleotides (Nuclei). $(B)$ pRNA is associated with heterochromatin. ChRIP assay monitoring the level of pRNA associated with chromatin that was precipitated with antibodies against Pol I, H3K4me3 (K4me3), H4K20me3 (K20me3), and TIP5. Data from the initial ChIP are presented as percent of input (light bars), and ChRIP data are presented as pRNA levels normalized to GAPDH mRNA levels (dark bars). (C) pRNA binding to chromatin requires sequences matching $\mathrm{T}_{0}$. Cells were depleted from pRNA by antisense LNA-DNA gapmers; retransfected with synthetic pRNA-205/-1, pRNA- $\Delta \mathrm{T}_{0}$, or pRNA-205/-127 (LS-127); and subjected to ChRIP using antibodies against H4K20me3. (D) pRNA interacts directly with $\mathrm{T}_{0}$. Triplex capture assay using native chromatin from cells that were transfected with biotin- and psoralen-labeled oligo \#1 or oligo \#4. After UV irradiation, DNA associated with the biotinylated oligos was captured with streptavidin-coated beads and analyzed by PCR using $0.2 \%$ of input and $1 \%$ of bound DNA. The bars on the right represent qPCR data of captured rDNA normalized to $\beta$-actin. $(E)$ Binding of TTF-I to its target site is competed by pRNA. EMSA showing binding of TTF-I $\Delta$ N185 to rDNA in the presence of 100 or 250 pmol (triangles) of a control oligo (\#1), an oligoribonucleotide comprising $\mathrm{T}_{0}$ (oligo\#4), pRNA-205/-1, or pRNA- $\Delta \mathrm{T}_{0}\left(\Delta \mathrm{T}_{0}\right)$. $(F)$ Formation of rDNA:pRNA structures impairs TTF-I binding to rDNA in vivo. ChIP showing the association of TTF-I with the rDNA promoter in the presence of a control RNA (Ctrl), pRNA-205/-1 (pRNA) or pRNA- $\Delta \mathrm{T}_{0}\left(\Delta \mathrm{T}_{0}\right) .(G)$ Binding of TTF-I and pRNA to rDNA is mutually exclusive. NIH3T3 cells were arrested at G1/S and released into $\mathrm{S}$ phase. Cells were harvested in mid $(5 \mathrm{~h})$ and late $\mathrm{S}$ phase $(8$ and $9 \mathrm{~h}$ after release), and TTF-I binding to rDNA was assayed by ChIP (dark bars). In parallel, chromatin-associated pRNA was determined by ChRIP using antibodies against H4K20me3 (gray bars). FACS analysis demonstrating S-phase progression is shown on the left $(n=2)$.

capture assay, using an oligoribonucleotide that carries a psoralen moiety at the $5^{\prime}$ end and a biotin residue at the $3^{\prime}$ end. The presence of psoralen allows fixation of the nucleic acid structure by UVA photoactivation without cross-linking proteins bound to DNA or RNA. A significant amount of rDNA was captured from cells that were transfected with a psoralen-labeled $\mathrm{T}_{0}$-containing oligoribonucleotide (Fig. 2D) or from isolated nuclei that were incubated with the $\mathrm{T}_{0}$ oligoribonucleotide (Supplemental Fig. S2A). This result demonstrates that the interaction of pRNA with $T_{0}$ is direct and not mediated by auxiliary proteins. Significantly, rDNA was captured with similar efficiency from samples treated with AccI, a restriction enzyme that cleaves within the $T_{0}$ element, demonstrating that the interaction with pRNA protected the AccI target site from digestion. Treatment with RNase VI or RNase $\mathrm{H}$ did not prevent the capture of rDNA (Supplemental Fig. S2B), indicating that the interaction of pRNA with $T_{0}$ is not brought about by dsRNA or by DNA:RNA duplexes.

To examine whether pRNA interferes with TTF-I binding to its target site, $\mathrm{T}_{0}$, we used EMSA to monitor TTF-I binding in the presence of synthetic pRNA or oligoribonucleotides. Binding of TTF-I to its cognate binding site was impaired in the presence of both oligo \#4 and pRNA-205/-1, whereas control oligo \#1 and the $\mathrm{T}_{0}$-deficient $\mathrm{mu}$ tant pRNA- $\Delta \mathrm{T}_{0}$ did not affect the interaction of TTF-I with rDNA (Fig. 2E). Consistently, transfection with oligo \#4 but not with a control oligo efficiently competed for TTF-I binding to the rDNA promoter in vivo (Supplemental Fig. S3C). Likewise, ectopic pRNA-205/-1, but not mutant pRNA- $\Delta \mathrm{T}_{0}$, decreased the occupancy of TTF-I at the rDNA promoter (Fig. 2F), indicating that the interaction of pRNA with $\mathrm{T}_{0}$ prevents binding of TTF-I to its cognate site.

NoRC-dependent silencing of rDNA occurs in late S phase; i.e., when silent copies of rRNA genes are replicated. To examine whether pRNA displaces TTF-I from its target site in vivo, we compared the chromatin association of pRNA and rDNA occupancy of TTF-I during S-phase progression (Fig. 2G). While the association of pRNA correlated with TIP5 binding to rDNA (Zhou et al. 2009), the binding of TTF-I and pRNA to $T_{0}$ were mutually exclusive. TTF-I was associated with chromatin at mid-S phase when no binding of pRNA was observed, whereas in late S phase the level of chromatin-associated pRNA increased and TTF-I binding decreased. The inverse association of TTF-I and pRNA with chromatin supports the view that the interaction of pRNA with $T_{0}$ either displaces TTF-I or prevents TTF-I binding to its target site. To examine whether pRNA sequences matching $T_{0}$ would have the potential to form a triple-stranded structure via Hoogsteen or reversed Hoogsteen base-pairing, we incubated radiolabeled rDNA fragments with tiled 20-nt RNA oligonucleotides and analyzed the formation of DNA:RNA triplexes by EMSA (Fig. 3A). The oligoribonucleotide comprising $\mathrm{T}_{0}$ (oligo \#4) but none of the other oligos retarded the mobility of the DNA fragment, suggesting that oligo \#4 interacted with rDNA, presumably by forming a triplex structure with the $T_{0}$ sequence. Given that triplex formation typically needs a homopurine strand in the DNA (Morgan and Wells 
A

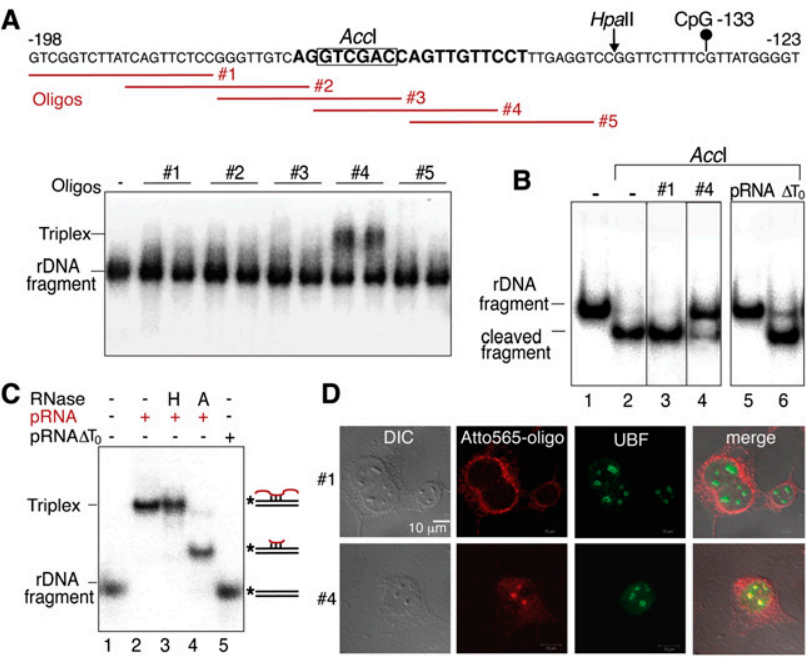

Figure 3. pRNA forms DNA:RNA triplex structures with $\mathrm{T}_{0} \cdot(A)$ Oligo \#4 forms a triplex structure with the rDNA promoter. The indicated pRNA oligos (\#1-\#5) were incubated with a ${ }^{32}$ P-labeled rDNA promoter fragment $(-205 /-140)$, and formation of DNA:RNA triplexes was monitored by EMSA. The sequence of the $5^{\prime}$-terminal part of the rDNA promoter, including the $\mathrm{T}_{0}$ sequence (bold) and adjacent sequences, as well as the position of tiled 20-nt oligoribonucleotides used (red), is displayed above. The AccI site (boxed), the $\mathrm{CpG}$ at -133 , and the HpaII site at -143 are marked. (B) Triplex formation impairs restriction endonuclease accessibility. (Lanes 1-4) A 5'-labeled rDNA fragment $(-205 /-140)$ was incubated with $10 \mathrm{U}$ of AccI for $30 \mathrm{~min}$ at room temperature in the absence or presence of oligo \#1 or oligo \#4, and cleavage was monitored by gel electrophoresis and autoradiography. In lanes 5 and 6, pRNA-205/-1 (pRNA) or mutant pRNA- $\Delta \mathrm{T}_{0}\left(\Delta \mathrm{T}_{0}\right)$ were used. $(C)$ rDNA:pRNA triplexes are resistant to RNase $\mathrm{H}$ digestion. (Lanes 2-4) Triplexes formed by incubating a labeled rDNA fragment with pRNA-205/-1 were treated with $30 \mathrm{U}$ of RNase $\mathrm{H}(\mathrm{H})$ or RNase A (A) for $30 \mathrm{~min}$ at room temperature and analyzed by EMSA. In lane 5, rDNA was incubated with mutant pRNA- $\Delta \mathrm{T}_{0}\left(\Delta \mathrm{T}_{0}\right)(D)$ An oligoribonucleotide comprising the $\mathrm{T}_{0}$ sequence is targeted to the nucleolus. Confocal images of permeabilized NIH3T3 cells after incubation with Atto565-labeled oligo \#1 or oligo \#4 (red) and immunostaining of UBF (green).

1968), this was an unexpected finding, as the $\mathrm{T}_{0}$ sequence is GC-rich but does not exhibit the typical features required for triplex formation. To validate the formation of a triplex structure comprising pRNA and $\mathrm{T}_{0}$, we performed restriction enzyme accessibility assays, taking advantage of associated RNA shielding the target DNA from cleavage (Gee et al. 1992). Cleavage by AccI was inhibited if the DNA was preincubated with oligo \#4 complementary to $\mathrm{T}_{0}$, but not with oligo \#1 (Fig. 3B). Protection was also observed with synthetic pRNA-205/-1 but not with pRNA- $\Delta \mathrm{T}_{0}$, underscoring the indispensability of $T_{0}$ for triplex formation. The amount of $p R N A$ required to protect DNA was 20-fold lower than that of oligo \#4, indicating that sequences adjacent to $T_{0}$ contribute to triplex formation. Treatment with RNase $\mathrm{H}$ did not affect the mobility of the DNA:RNA complex, ruling out the possibility that the shift in electrophoretic mobility was due to the formation of DNA:RNA heteroduplexes. Again, no triplex formation was observed with pRNA- $\Delta \mathrm{T}_{0}$ (Fig. $3 \mathrm{C})$, reinforcing the requirement of pRNA base-pairing with $\mathrm{T}_{0}$.

To demonstrate that pRNA is targeted to nucleolar chromatin by interacting with $\mathrm{T}_{0}$ in vivo, we incubated permeabilized cells with fluorescent pRNA oligos and monitored their cellular localization. As shown in Figure 3D, oligo \#4 but not oligo \#1 localized to nucleoli, emphasizing the importance of $\mathrm{T}_{0}$ for recruiting pRNA to the nucleolus. Incubation with RNase $\mathrm{H}$ did not release oligo \#4 from nucleoli, while preformed DNA:RNA heteroduplexes were cleaved and dispersed throughout the cell (Supplemental Fig. S4A). These data support the notion that pRNA forms a DNA:RNA triplex rather than a DNA:RNA duplex, and triplex formation retained oligo \#4 in nucleoli.

To examine whether pRNA recruits methyltransferase(s) to rDNA, we artificially raised the level of cellular pRNA by transfecting synthetic pRNA, and monitored rDNA occupancy of TIP5, DNMT1, and DNMT3b. While not affecting NoRC occupancy, ectopic pRNA triggered substantial enrichment of DNMT3b at the rDNA promoter (Fig. 4A). To verify that pRNA-dependent hypermethylation is due to targeting of de novo DNMT(s) to rDNA, we transfected pRNA-205/-1 into cells that were depleted of DNMT3a or DNMT3b, and monitored changes in rDNA promoter methylation. Ectopic pRNA increased DNA methylation in cells depleted of DNMT3a, but did not affect DNA methylation in cells depleted of DNMT3b (Fig. 4B), indicating that pRNA targets DNMT3b to rDNA. Consistently, Southwestern blot and pull-down assays demonstrate that DNMT3b exhibits a pronounced preference for DNA:RNA triplexes compared with duplex rDNA (Fig. 4C,D). All three DNMTs also bind RNA (Supplemental Fig. S5); however, only

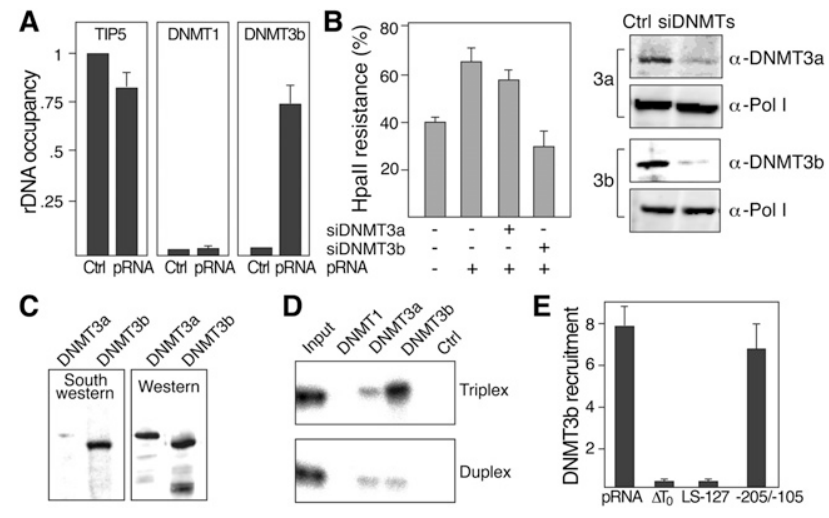

Figure 4. pRNA recruits DNMT3b to rDNA. $(A)$ Ectopic pRNA enhances rDNA occupancy of DNMT3b. ChIP showing the association of Myc-tagged DNMT1 and DNMT3b with the rDNA promoter in NIH3T3 cells transfected with control RNA (Ctrl) or pRNA-205/-1 (pRNA). (B) Knockdown of DNMT3b abolishes pRNA-dependent DNA methylation. Data of methylation-sensitive PCR in DNMT3a- and DNMT3b-depleted cells that have been transfected with synthetic pRNA. The Western blots on the right show the knockdown of DNMTs. $(C)$ Southwestern blot. Similar amounts of membrane-bound DNMT3a and DNMT3b were incubated with radiolabeled DNA:RNA triplexes, and bound triplexes were visualized by autoradiography. The Western blot shows the amount of immobilized DNMTs. (D) Dnmt3b preferentially binds DNA:RNA triplexes. Pull-down experiment using immobilized GFP-tagged DNMT1, DNMT3a, DNMT3b, or control beads and radiolabeled rDNA:pRNA triplexes (top) or rDNA duplexes (bottom). (E) The part of pRNA corresponding to $\mathrm{T}_{0}$ is essential for recruitment of DNMT3b to the rDNA promoter. ChIP of Myctagged DNMT3b from cells that were transfected with ectopic pRNA-205/-1(pRNA), pRNA- $\Delta \mathrm{T}_{0}\left(\Delta \mathrm{T}_{0}\right)$, pRNA/LS-205/-127 (LS127), or pRNA-205/-105 (-205/-105). 
DNMT3b shows a preference for DNA:RNA triplexes. Of note, the fact that DNMT3b but not DNMT3a is attracted by triplex structures suggests a putative selection mechanism of target genes for DNMT3b, increasing the diversity and potential of epigenetic regulation of gene expression.

To identify the part of pRNA that mediates DNMT3b recruitment, we analyzed DNMT3b binding to rDNA after transfection of NIH3T3 cells with wild-type and mutant pRNAs. Notably, while ectopic pRNA enhanced DNMT3b occupancy at the rDNA promoter, pRNA- $\Delta \mathrm{T}_{0}$ and pRNA-127/-39 did not affect DNMT3b binding. However, pRNA-205/-105 lacking the stem-loop structure required for TIP5 binding was as efficient as full-length pRNA in mediating DNMT3b recruitment (Fig. 4E). This result demonstrates that pRNA is capable of recruiting DNMT(s) to the rDNA promoter, and its ability to form a triplex with the $T_{0}$ element is a prerequisite for pRNAdirected rDNA methylation (Supplemental Fig. S6). Together, these findings suggest an RNA-based strategy for epigenetic programming, implying that ncRNAs can act as selective ligands for chromatin-modifying enzymes.

The idea that ncRNA would be well adapted for regulatory roles is not new. Almost 50 years ago, Jacob and Monod (1961) reasoned that base complementarity would allow RNA to interact highly specifically with other nucleic acid sequences. They proposed that some genes produce regulatory RNAs that interact by base-pairing with specific sequences. The potential of RNA to bind to complementary DNA sequences has led to the proposal that RNA plays an important "guiding" role in the establishment and transmission of chromatin states. Although the in vivo existence of DNA:RNA triplexes and their role in epigenetic programming still need to be validated, our study provides novel mechanistic insights into the mode of action of ncRNA in chromatin-based processes, implicating a direct and possibly widespread role of RNA:DNA structures in epigenetic regulation. In support of this view, triple-helix target sites were found to be overrepresented at human gene promoters, highlighting the potential of naturally occurring hydrogen bond interactions of ncRNA with the major groove of DNA to control gene expression (Goñi et al. 2004; Belotserkovskii et al. 2007). A triplex-mediated mechanism of DNA methylation and gene silencing is particularly intriguing considering that the vast majority of the transcriptional output of the human genome represents ncRNA (Prasanth and Spector 2007; Bühler 2009; Mattick 2009). Therefore, ncRNAs can provide unique sequence specificity via triplex formation and may function as binding platforms for chromatin-modifying enzymes without the need of adaptor proteins. Of note, an ncRNA-mediated targeting mechanism of DNMTs would have the potential to act on virtually any gene that is silenced by DNA methylation.

\section{Materials and methods}

Antibodies, plasmids, and standard methods are described in the Supplemental Material. Data are represented as mean \pm SD values from several independent experiments.

\section{Triplex formation, triplex capture, and triplex-binding assays}

A total of 0.5 pmol of a ${ }^{32}$ P-labeled rDNA fragment (from -205 to -140 ) was incubated with 6 pmol of pRNA in $10 \mathrm{mM}$ Tris- $\mathrm{HCl}(\mathrm{pH} 7.4), 25 \mathrm{mM}$ $\mathrm{NaCl}, 10 \mathrm{mM} \mathrm{MgCl} 2$, and $10 \%$ glycerol for $2 \mathrm{~h}$ at room temperature.
Triplex formation was monitored by electrophoresis on $8 \%$ polyacrylamide gels containing $10 \mathrm{mM} \mathrm{MgCl}_{2}$. For triplex capture assays, isolated nuclei were incubated with $10 \mu \mathrm{M}$ biotinylated RNA oligonucleotides for $90 \mathrm{~min}$ at $30^{\circ} \mathrm{C}$ in $10 \mathrm{mM}$ Tris- $\mathrm{HCl}(\mathrm{pH} 7.4), 25 \mathrm{mM} \mathrm{NaCl}$, and $10 \mathrm{mM}$ $\mathrm{MgCl}_{2}$ (Besch et al. 2004). Alternatively, NIH3T3 cells were transfected with 140 pmol of biotin-labeled pRNA-205/-105 or 200 pmol of biotinylated oligoribonucleotides and harvested after $48 \mathrm{~h}$. Cells were crosslinked with $1 \%$ formaldehyde (10 min for cells, $5 \mathrm{~min}$ for nuclei) and lysed in $20 \mathrm{mM}$ Tris-HCl (pH 7.4), $250 \mathrm{mM} \mathrm{NaCl}, 2$ mM EDTA, 2 mM EGTA, and $1 \%$ Triton X-100. After sonication, biotinylated RNA was captured with streptavidin-coated magnetic beads that were blocked with $0.25 \mathrm{mg} /$ $\mathrm{mL}$ herring sperm DNA, $2 \mathrm{mg} / \mathrm{mL}$ BSA, $1 \mathrm{mg} / \mathrm{mL}$ tRNA, $1 \%$ gelatin, and $0.2 \mathrm{mg} / \mathrm{mL}$ chicken egg white albumin. DNA:RNA complexes were eluted with $10 \%$ Chelex (Bio-Rad), digested with proteinase $\mathrm{K}$, and analyzed by quantitative PCR (qPCR). If biotin- and psoralen-modified RNA oligonucleotides were used, cells or nuclei were irradiated for $5 \mathrm{~min}$ with UVA $(365 \mathrm{~nm})$ before lysis. As controls, lysates were treated with $2 \mathrm{U}$ of RNase V1 (Ambion), $15 \mathrm{U}$ of RNase H (BioLabs), or $15 \mathrm{U}$ of AccI (Promega) for $20 \mathrm{~min}$ at $30^{\circ} \mathrm{C}$. Genomic DNA was digested with EcoRI and incubated with streptavidin beads. After elution with $0.1 \%$ SDS, captured DNA was digested with proteinase $\mathrm{K}$ and analyzed by qPCR. Data are presented as the ratio of captured rDNA versus GAPDH or $\beta$-actin.

To monitor binding of DNMTs, HEK293T cells overexpressing GFPtagged DNMT1, DNMT3a, and DNMT3b were lysed in $700 \mu \mathrm{L}$ of L-buffer (20 mM Tris- $\mathrm{HCl}$ at $\mathrm{pH} 7.4,300 \mathrm{mM} \mathrm{NaCl}, 2 \mathrm{mM}$ EDTA, $2 \mathrm{mM}$ EGTA, $1 \%$ Triton X-100, $0.5 \mathrm{mM}$ PMSF, $1 \mathrm{mg} / \mathrm{mL}$ aprotinin, $5 \mathrm{mg} / \mathrm{mL}$ leupeptin, $1 \mathrm{mg} / \mathrm{mL}$ pepstatin A). GFP-DNMTs, bound to GFP-Trap were incubated for $30 \mathrm{~min}$ at $4^{\circ} \mathrm{C}$ with ${ }^{32} \mathrm{P}$-labeled DNA duplexes or DNA:RNA triplexes. Retained nucleic acids were visualized by electrophoresis on $8 \%$ polyacrylamide gels and autoradiography.

\section{Acknowledgments}

We thank T. Kouzarides for the expression vector encoding DNMT1; C.-L. Hsieh for DNMT3b; H. Leonhardt for GFP-DNMT1, GFP-DNMT3a, and GFP-DNMT3b; E. Kremmer for anti-Myc antibodies; and Nina Schmitt and Jana Krug for valuable assistance. This work has been supported by a European Research Council Advanced Grant, the Deutsche Forschungsgemeinschaft, and the Fonds der Chemischen Industrie.

\section{References}

Belotserkovskii BP, De Silva E, Tornaletti S, Wang G, Vasquez KM, Hanawalt PC. 2007. A triplex-forming sequence from the human c-MYC promoter interferes with DNA transcription. I Biol Chem 282: 32433-32441.

Bernstein E, Allis CD. 2005. RNA meets chromatin. Genes Dev 19: 16351655.

Besch R, Giovannangeli C, Schuh T, Kammerbauer C, Degitz K. 2004. Characterization and quantification of triple helix formation in chromosomal DNA. J Mol Biol 341: 979-989.

Bühler M. 2009. RNA turnover and chromatin-dependent gene silencing. Chromosoma 118: 141-151.

Gee JE, Blume SW, Snyder RC, Ray R, Miller DM. 1992. Triplex formation prevents SP1 binding to the dihydrofolate reductase promoter. J Biol Chem 267: 11163-11167.

Goñi JR, de la Cruz X, Orozco M. 2004. Triplex-forming oligonucleotide target sequences in the human genome. Nucleic Acids Res 32: 354360 .

Goodrich JA, Kugel JF. 2009. From bacteria to humans, chromatin to elongation, and activation to repression: The expanding roles of noncoding RNAs in regulating transcription. Crit Rev Biochem Mol Biol 44: $3-15$.

Grummt I, Kuhn A, Bartsch I, Rosenbauer H. 1986. A transcription terminator located upstream of the mouse rDNA initiation site affects rRNA synthesis. Cell 47: 901-911.

Jacob F, Monod J. 1961. Genetic regulatory mechanisms in the synthesis of proteins. I Mol Biol 3: 318-356.

Mattick JS. 2009. The genetic signatures of noncoding RNAs. PLoS Genet 5: e1000459. doi: 10.1371/journal.pgen.1000459.

Mayer C, Schmitz K-M, Li J, Grummt I, Santoro R. 2006. Intergenic transcripts regulate the epigenetic state of rRNA genes. Mol Cell 22: 351-361. 
Mayer C, Neubert M, Grummt I. 2008. The structure of NoRC-associated RNA is crucial for targeting the chromatin remodelling complex NoRC to the nucleolus. EMBO Rep 9: 774-780.

Morgan AR, Wells RD. 1968. Specificity of the three-stranded complex formation between double-stranded DNA and single-stranded RNA containing repeating nucleotide sequences. J Mol Biol 37: 63-80.

Prasanth KV, Spector DL. 2007. Eukaryotic regulatory RNAs: An answer to the 'genome complexity' conundrum. Genes Dev 21: 11-42.

Santoro R, Grummt I. 2001. Molecular mechanisms mediating methylation-dependent silencing of ribosomal gene transcription. Mol Cell 8: 719-725.

Santoro R, Li J, Grummt I. 2002. The nucleolar remodeling complex NoRC mediates heterochromatin formation and silencing of ribosomal gene transcription. Nat Genet 32: 393-396.

Strohner R, Nemeth A, Jansa P, Hofmann-Rohrer U, Santoro R, Längst G, Grummt I. 2001. NoRC-A novel member of mammalian ISWIcontaining chromatin remodeling machines. EMBO J 20: 4892-4900.

Zhou Y, Grummt I. 2005. The PHD finger/bromodomain of NoRC interacts with acetylated histone H4K16 and is sufficient for rDNA silencing. Curr Biol 15: 1434-1438.

Zhou Y, Santoro R, Grummt I. 2002. The chromatin remodeling complex NoRC targets HDAC1 to the ribosomal gene promoter and represses RNA polymerase I transcription. EMBO J 21: 4632-4640.

Zhou Y, Schmitz KM, Mayer C, Yuan X, Akhtar A, Grummt I. 2009. Reversible acetylation of the chromatin remodelling complex NoRC is required for non-coding RNA-dependent silencing. Nat Cell Biol 11: $1010-1016$ 


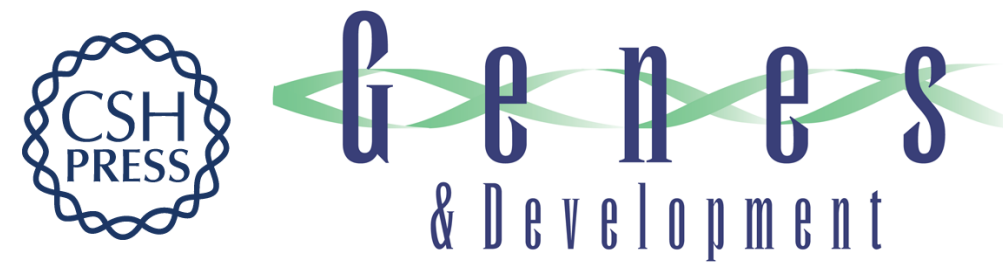

\section{Interaction of noncoding RNA with the rDNA promoter mediates recruitment of DNMT3b and silencing of rRNA genes}

Kerstin-Maike Schmitz, Christine Mayer, Anna Postepska, et al.

Genes Dev. 2010, 24:

Access the most recent version at doi:10.1101/gad.590910

Supplemental http://genesdev.cshlp.org/content/suppl/2010/10/13/24.20.2264.DC1
Material

References This article cites 20 articles, 6 of which can be accessed free at:

http://genesdev.cshlp.org/content/24/20/2264.full.html\#ref-list-1

License

Email Alerting Receive free email alerts when new articles cite this article - sign up in the box at the top

Service

right corner of the article or click here.

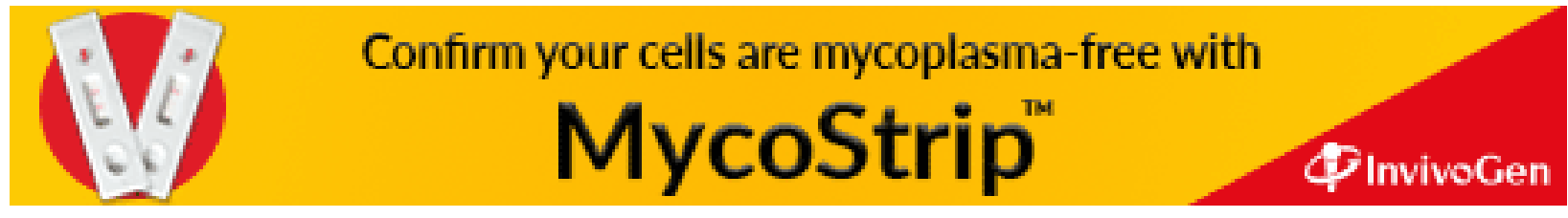

